\title{
Toward a Psychophysiology of Expertise
} Focal Magnetic Gamma Bursts as a Signature of
Memory Chunks and the Aptitude of Chess Players

\author{
Ognjen Amidzic, Hartmut J. Riehle, and Thomas Elbert
}

University of Konstanz, Germany

\begin{abstract}
Gamma-band activity (GBA) bursts have been viewed as a signature for ignitions in localized Hebbian cell assemblies and are thought to indicate active memory. Using whole-head magnetoencephalography, we recorded focal bursts of GBA during chess playing. Unlike highly skilled chess grandmasters, with amateur chess players focal gamma bursts prevailed in deeper structures in the region of the medial temporal lobes. This observation is consistent with the interpretation of memory formation in amateur chess players. For the frontal and parietal cortex the relationship was reversed, with more frequent gamma bursts found in chess grandmasters, indicating that the retrieval of chunks from expert memory is based on the recruitment of these neocortical areas. The results suggest the possibility that time-dependent reorganization during the formation of expert memory can be studied in humans and support the theory that the medial temporal lobe and hippocampal formation play a transitional role during the creation of expert memory in the neocortex.
\end{abstract}

Keywords: expert memory, chess, giftedness, aptitude, chunks, gamma band

\section{Introduction}

The game of chess offers a model task environment for the study of basic cognitive processes: perception, memory, and problem solving. Chess has also enjoyed a privileged position in artificial intelligence research, as a model domain for exploratory search and evaluation processes. Probably the most important tool developed for research on chess playing is Elo's (Elo, 1978) chess-skill rating scale. Elo's interval-level rating scale provides chess researchers with a valid measurement device; a unique opportunity for the study of individual differences unrivaled in other areas of expertise research. A number of models have been suggested to account for expertise in chess players (Chase \& Simon, 1973a,b; Ericsson \& Knitsch, 1995; Gobet \& Simon, 1996a,b; Holding, 1992; Holding \& Reynolds, 1982). In a comparison of the four most influential theories, Gobet (1998) concludes that the chunking theory (Chase \& Simon, 1973a,b) is consistent with data including the recall of random and distorted positions, recall with very short presentation time, and interference studies. Chunking theory suggests that expert memory is based on a large database of chunks in long-term memory. A typical chess grandmaster may have practiced and studied for at least 10 years to learn more than 100,000 positions (memory chunks). Consequently, grandmasters can "recognize" the key elements in a problem situation much more rapidly than amateur players. However, experts differ not only with respect to amount of knowledge (quantitatively), but also with respect to its organization (qualitatively) (Gobet, 1998). Gobet and Simon's template theory consequently incorporates high-level processing elements such as structuring knowledge and planning that assist in accessing the respective chunks. This theory provides the best account of the empirical evidence derived from behavioral data (Gobet, 1998).

A neurophysiological test of chunking-based theories requires the detection and localization of the activation of single chunks. It is widely believed that memory chunks may be represented in Hebbian cell assemblies, i.e., in neuronal connectivity that forms in response to the frequent coactivation of neurons. Hebb's prediction that learning strengthens synaptic connectivity through long-term potentiation (LTP) has recently been demonstrated in the neocortex (Rioult-Pedotti, Friedman, \& Donoghue, 2000). Strong internal connectivity within a cell assembly assures that it will be fully activated when a sufficient share of its elements is activated (ignited). Assuming that once activated, a cell assembly will remain active for a short period of time and that it contains at least some 10,000 neurons, its activation will initiate a firing sequence that produces a measurable magnetoencephalographic (MEG) signal (Elbert, 1998). While Hebbian assemblies may be widespread across cortical regions, the neuroanatomical examination of neocortical connectivity suggests that cell assemblies are often confined to a focal brain region (Brai- 
tenberg \& Schüz, 1991). An equivalent current dipole (ECD) model provides an excellent approximation of focal brain activity (Elbert, 1998). Therefore, the localization of ECDs in the gamma-band range may be used as a tool to localize the triggering of focal neuronal cell assemblies that include a sufficiently large number of elements. The gamma band has been used here as there is little or no overlap from unspecific widespread cortical synchronization, e.g., alpha or delta (for an overview of gamma-band studies and their interpretation see, e.g., Pulvermüller, Keil, \& Elbert, 1999; Tallon-Baudry \& Bertrand, 1999). We suggest that memory chunks are represented by the triggering of exactly such cell assemblies that may fire at any frequency from 1 to $100 \mathrm{~Hz}$. Therefore, the activation of chunks will - on the average - trigger activity in a corresponding brain area that is also in the gamma band. The center of this focal area can be tracked by means of magnetic source imaging if, and only if, during exactly the same period there is little ongoing activity in the surrounding brain region that has the same frequency characteristics as the one to be localized. In this case, the ECD model will provide an excellent fit, whereas a good fit is very unlikely if several generator sources overlap in time. Scanning the single unaveraged MEG traces filtered in the gamma-band range for time points at which the ECD-model fit is perfect will reveal bursts of focal neuronal activity and allow for its localization.

Evidence from brain imaging (Bontempi, Laurent-Demir, Destrade, \& Jaffard, 1999) and lesion studies (Squire, 1992; Teng, 1999) suggests that the hippocampal formation and related structures play a key role in the initial formation of long-term memory, but may be of lesser importance for the retrieval of memories more remote in time (Bontempi et al., 1999; Squire, 1992; Teng, 1999). Here we show that during chess playing the distribution of focal brain activity in the gamma-band range differs between chess grandmasters and amateur players. Grandmasters display gamma bursts in the neocortex when analyzing chess moves, whereas in amateur players activity predominates in the region of the medial temporal lobe and neighboring structures (Amidzic, Riehle, Fehr, Wienbruch, \& Elbert, 2001).

A short note, describing some data has been published previously (Amidzic et al., 2001): This paper describes the procedures of the methods and techniques, so that replication is possible. We also describe the outcome in more detail, in particular, we present data about the brain laterality during the cognitive task of chess playing, which was not included in the original short communication.

\section{Methods}

The 20 players investigated in this study had more than 10 years of tournament and training practice. According to their self-reports, they were highly motivated to increase their performance skills but had reached a plateau Elo quotient with yearly changes of less than 40 Elo points in either direction. Ten professional players (grandmasters) scored between 2400 and 2600 Elo points. The amateur players in the study ranked 1700 and above on the Elo scale. The age of the 20 male chess players (mean 42.5 yrs., $S D$ 13.7; 19 right-handed) was unrelated to Elo. MEG refers to the measurement of magnetic fields outside the brain that originate from intracellular current flow within the brain. Use of digitization hardware and software (4D Neuroimaging) made possible the location in 3D-space and the collection of information about head shape relative to the sensor, and the computation of the relationship between the head and the Cartesian coordinate system of the sensor, which is the necessary assumption for deep source localization.

MEG data were obtained while subjects played against a computer. During play, MEG was measured using a 148channel whole-head magnetometer (WHS 2500, 4D Neuroimaging) in a magnetically shielded room. The magnetic sensing devices (magnetometers) were spaced $28 \mathrm{~mm}$ apart and were arranged in a uniformly distributed array on the inside surface of a helmet. In order to eliminate ocular artifacts, eye movements (EOG) were recorded with electrodes above and below the right eye and from both outer canthi. Environmental noise in the signal channels was also measured with remote detectors (referent channels) placed at a distance from the physiological signal generators and was subtracted from the signal channel outputs. For the acquisition, source analysis, filtering and noise reduction, standard 4D Neuroimaging software was used.

Data were recorded with a passband of $0.1-200 \mathrm{~Hz}$ and sampled at a rate of $678.15 \mathrm{~Hz}$. Following a move by the computer program, the filtered single MEG segments (epochs: $2 \mathrm{~s}$ prestimulus and $5 \mathrm{~s}$ poststimulus) were screened without averaging for the appearance of local gamma bursts, defined as the number of dipoles/voxel that could be fitted to the activity in the $20-40 \mathrm{~Hz}$ band range. This dipole density was calculated across all artifact-free segments for each subject. The mean number of epochs was 44 (SD 25). Responses were filtered from 20 to $40 \mathrm{~Hz}$ (with an additional notch at $50 \mathrm{~Hz}$ ). Dipole fits (single equivalent dipole in a homogeneous sphere) were calculated for standard 4D neuroimaging channel selections (148 channels) at each time-point of the selected raw epochs (not averaged epochs) following a move by the computer. Only when the goodness-of-fit was greater than 0.90 were data accepted for further analyses. In case of averaged event-related fields (ERF), the original data and the field patterns should provide a typical pattern. In this study, however, we did not rely on ERF, but calculated ECDs by using a regional single dipole model to fit the digitally filtered but nonaveraged MEG traces for every sampling point of the $678.15 \mathrm{~Hz}$ sampling rate. Figure 1A shows the example of gamma bursts in a single epoch. As there is no ERF in the filtered data, no typical patterns could be delineated, and the example is given to verify the reliability of the applied methodological approach. Figure 1B shows the example of a contour plot based on raw MEG data where a high goodness-of-fit appears and the dipole fit is in the upper right.

The number of dipoles in each brain voxel was deter- 


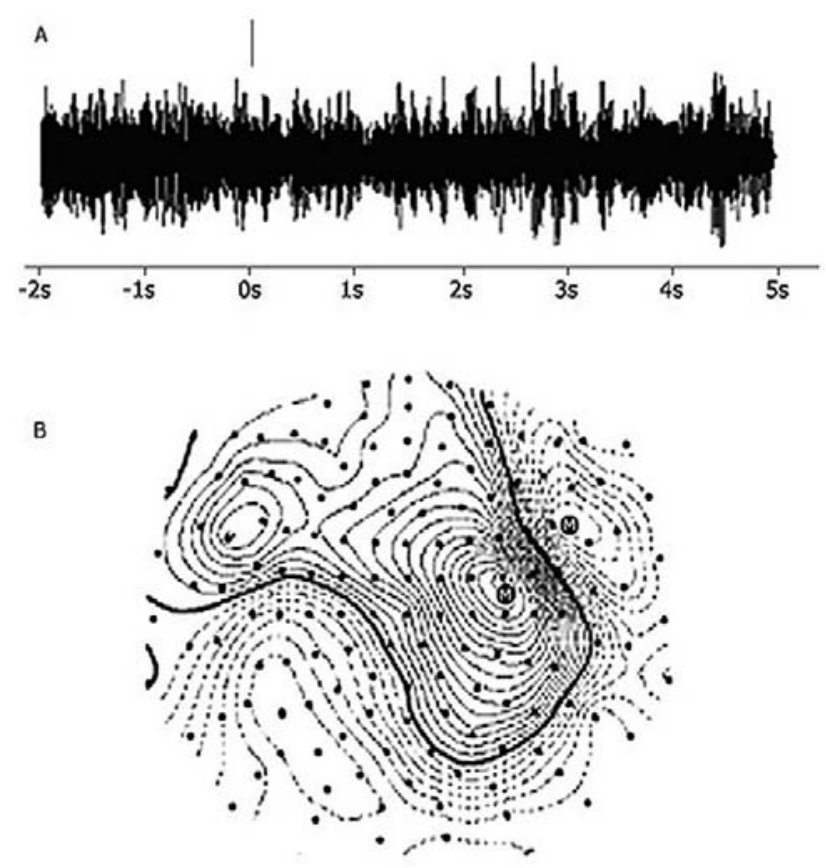

Figure 1. An example of gamma bursts in a single epoch (A) with no typical ERF patterns. High goodness of fit and the dipole fit on the right side of the contour plot based on raw MEG data (B). mined for each epoch, and then for each $2 \times 2 \times 2 \mathrm{~cm}$ voxel separately. The number of dipoles with goodness-if-fit $>$ 0.90 was then counted within the $2 \times 2 \times 2 \mathrm{~cm}$ voxels, representing a matrix in the Cartesian coordinate system (Figure 2) defined by a y-axis through the left (LPA) and right preauricular (RPA) points and an x-axis that crosses the center point of the $y$-axis (origin) and the nasion. The $\mathrm{z}$-axis leads orthogonally to the $\mathrm{x} / \mathrm{y}$-plane through this origin. The dipole density (in \%) was determined by dividing the number of dipoles in each voxel by the total number of dipoles found for the subject in any region and by multiplying the result by 100 (for a more detailed description of dipole density measurements, see Elbert, 1998; Rockstroh et al., 2004; Vieth, Kober, \& Grummich, 1996). The root mean square (RMS) ranged from $0.4 \mathrm{fT}$ to $740 \mathrm{fT}$. The lateralization coefficient (right - left)/(right + left) was computed for the dipole densities calculated separately across each hemisphere and, with the range -1 to 1 , served to determine group differences in hemispheric lateralization.

\section{Results}

Figure 2 illustrates tomograms of dipole density. Examination of single slices indicates - in amateur players (Figure 2B), but not in grandmasters (Figure 2C) - pronounced di-
A

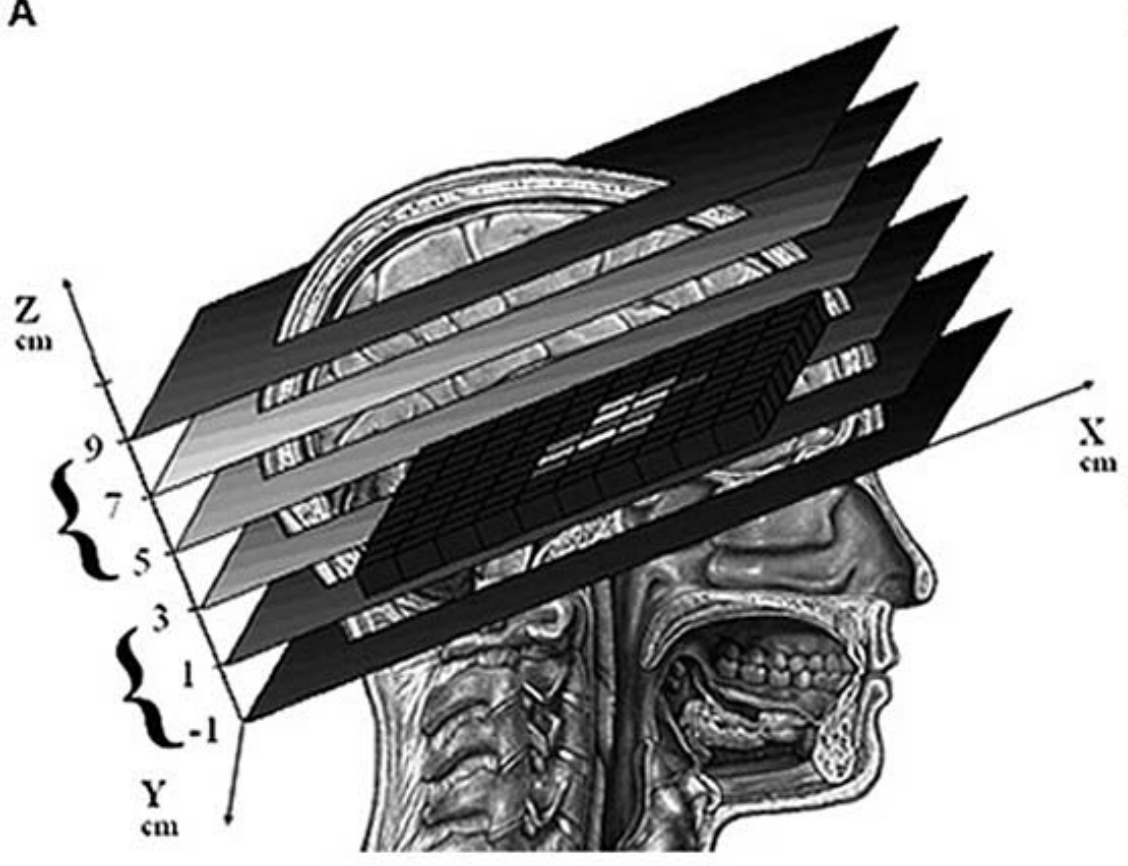

B

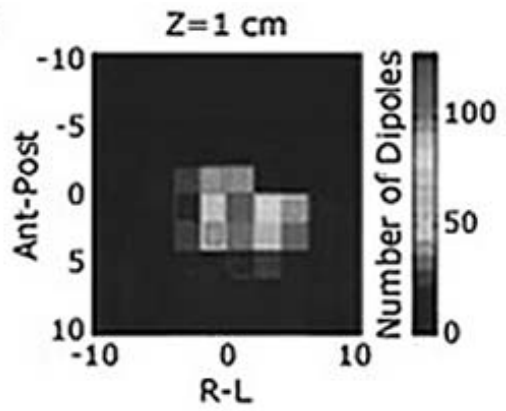

C

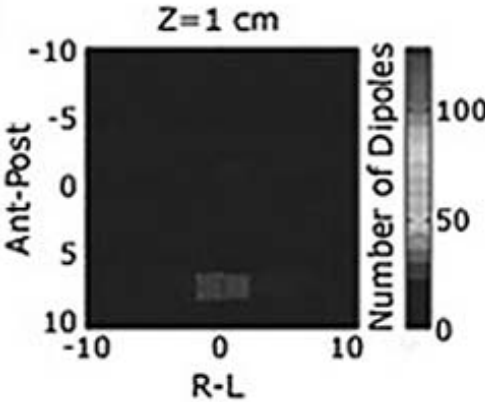

Figure 2. The coordinate system is defined by a y-axis through the left (LPA) and right preauricular (RPA) points, an $\mathrm{x}$-axis that crosses the center point of the $\mathrm{y}$-axis (origin) and the nasion, and the $\mathrm{z}$-axis, which leads orthogonally to the $\mathrm{x} / \mathrm{y}$-plane through this origin. Dipole density is plotted in a number of slices along the $\mathrm{z}$-axis. Goodness of fit GOF $>90 \%$ was tested for every dipole localized for every sampling point and placed in a voxel. The voxels represent a matrix in the Cartesian sensor system (A). Examination of single slices indicates - in amateur players (B), but not in grandmasters (C) - pronounced dipole density in the region of the perirhinal and entorhinal cortex, hippocampus, and related structures. 


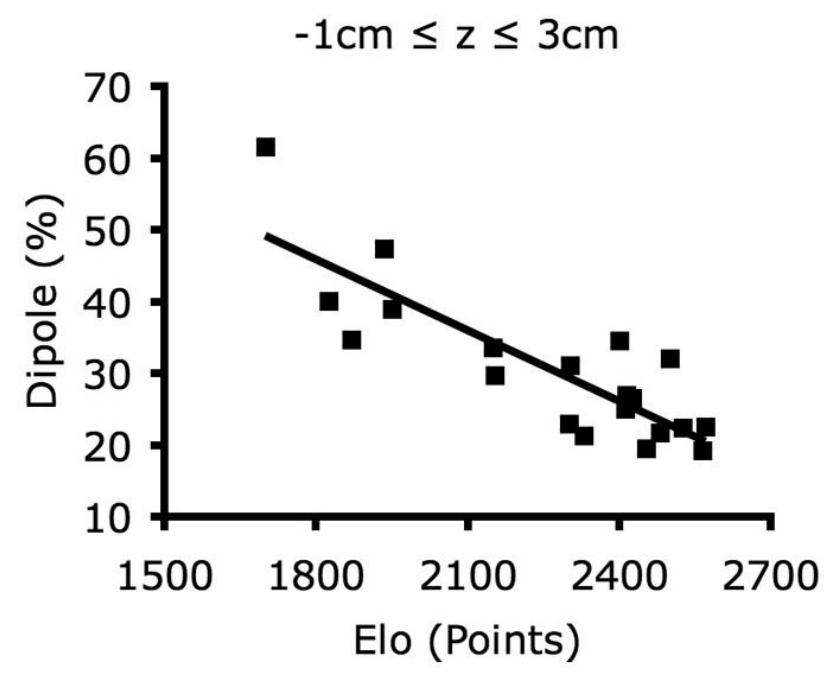

Figure 3. A strong correlation $(r=-0.84)$ between the relative number of dipoles and Elo found in levels -1 to $3 \mathrm{~cm}$ along the $\mathrm{Z}$ axis include the medial temporal lobe, hippocampus, and related structures. In contrast, the correlation was reversed $(r=0.85)$ for slices $5 \mathrm{~cm} \leq \mathrm{z} \leq 9 \mathrm{~cm}$, which include exclusively neocortical areas. pole density in the region of the perirhinal and entorhinal cortex, hippocampus, and related structures. A strong negative correlation $(r=-0.84)$ between the relative number of dipoles and Elo was found in levels $-1 \mathrm{~cm} \leq \mathrm{z} \leq 3 \mathrm{~cm}$ (Figure 3), which include the medial temporal lobe, hippocampus, and related structures. In contrast, the correlation was reversed for slices $5 \mathrm{~cm} \leq z \leq 9 \mathrm{~cm}$, which include exclusively neocortical areas $(r=0.85)$. In some amateur players there was also enhanced dipole density in the cerebellar regions, but a much larger number of subjects or epochs would be needed to reach a statistically valid conclusion. There was no significant correlation between the dipole density measures and age $(r \leq 0.02$ and $r \leq-0.03)$. Nor was there a relationship between the length, complexity or result of the game and the Elo score. Figure 4 illustrates how the correlation between chess expertise and the number of gamma bursts flips along the z-axis, i.e., from inferior to superior tomograms. These pronounced differences in the magnitude and distribution of brain activity during chess playing point to different mechanisms of brain processing and functional brain organization between grandmasters and amateurs. In grandmasters, up to $80 \%$ of the dipoles are localized in cortical areas of the frontal,
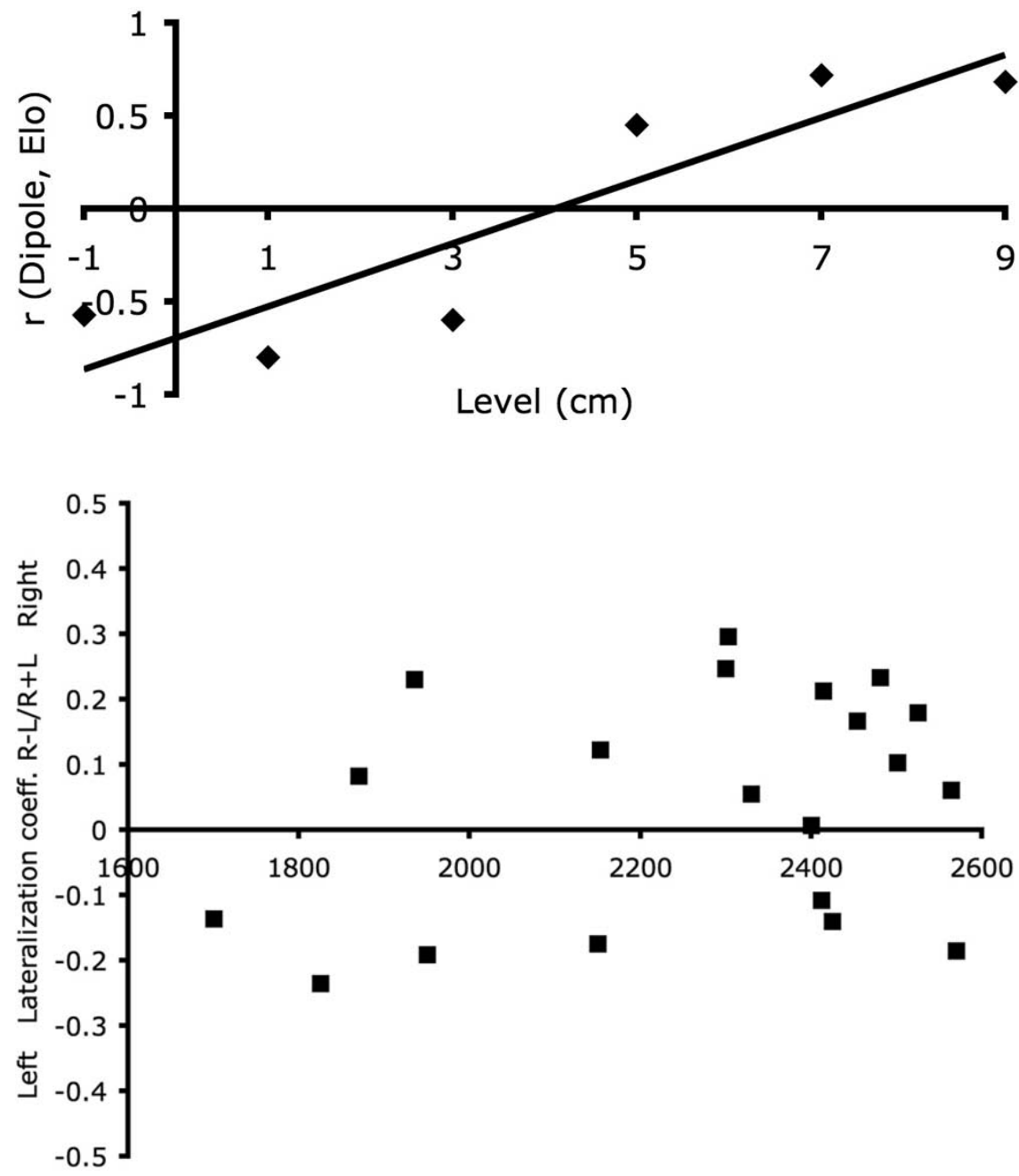

Figure 4. The correlation coefficients between the number of active gamma dipoles and Elo computed for each $\mathrm{x} / \mathrm{y}$-slice are plotted as a function of the location of the $\mathrm{x} / \mathrm{y}$-slices along the z-axis. The relationship ( $r=0.9)$ suggests that players with a strong Elo have activity predominantly in the higher slices through the neocortex, whereas with amateur players activity is centered in inferior regions.

Figure 5. Lateralization during chess playing: There were no differences in the distribution of dipoles (GOF > $90 \%$ in the 20 to $40 \mathrm{~Hz}$ range) between the hemispheres and no correlation between the lateralization coefficient and the Elo. 
parietal, and occipital lobes. In contrast to professionals, in amateur players activity occurred predominantly in deeper structures, mainly in the region of the medial temporal lobe. Figure 5 demonstrates that there were no lateralization differences between the players. The allocation of the dipoles in both hemispheres is almost identical.

\section{Discussion}

The dipole density analysis procedure has been validated, successfully applied, and presented by a number of groups particularly for slower frequency bands (e.g., Elbert, 1998; Fernandez et al., 2002; Lewine \& Orrison, 1995; Meinzer et al., 2004; Vieth et al., 1996; Wienbruch et al., 2003). It should be noted that widespread sources are radially oriented with respect to the head's surface and, thus, go largely undetected in MEG recordings. In other words, MEG acts like a filter extracting focal activities. Unlike EEG, MEG is not affected by volume conduction, and, therefore, mainly sources near the sensors contribute to the signal. If there is more than one source active at a given moment in time, the goodness-of-fit will decrease and was discarded in the present analysis, i.e., these sources are not modeled by the present analysis. In addition, noise reduction, EOG recording, and extracting the environmental noise from the physiological signal generators will provide high quality data making it possible to represent the deep temporal lobe and related structures.

Our findings suggest that activation occurs in the medial temporal lobe, hippocampus, and related structures of amateur players. Lesions in these structures impair recent memory while leaving remote memory intact (Squire, 1992; Zola-Morgan \& Squire, 1990). Grandmasters, who produce small (or few) gamma bursts in these structures, obviously seem to rely more on remote than on recent memory. The chunking theory of memory states that the number and nature of chunks that chess experts can hold in long-term memory can be used to predict chess performance (Chase \& Simon, 1973a,b). The present results suggest that the activation of expert memory chunks produces focal gamma-band activity that may occur in various neocortical areas. A precise anatomical location would require a better signal-to-noise ratio than that available in single trials and also a superposition with individual MRI slices. Nevertheless, the dramatic macroscopic differences between activation in grandmasters and amateurs in response to the computer's moves supports the view that professional players recall stored information from long-term memory, activating cell assemblies in the neocortex, while amateur players primarily encode new information, a task that would activate medial temporal lobe structures and the hippocampus rather than predominantly neocortical areas. It is possible that the medial temporal lobe and the hippocampal formation play only a transitional role during the creation of expert memory in the neocortex (Amidzic et al.,
2001). High-level processing, such as structuring knowledge and planning, which assists in accessing a systematic sequence of chunks (Gobet \& Simon 1996a,b), would only appear in the present analysis if confined to brain regions small enough to be fitted by a single dipole. Some of the dipoles presently counted in the neocortex may be related to such activity. However, the systematic chain-like activation of chunks is more likely to require widespread networks that if activated would not appear in the present analysis. An analysis of the temporal dynamics of the sequence of gamma bursts would be required to track the higher-level processing. Finally, it should be noted that the present analysis examines very brief episodes of focal neural activation, which are unlikely to produce a sufficient signalto-noise ratio in the inert hemodynamic measures. Thus, it might be useful to add this type of analysis to the tools currently available for brain imaging. It is possible that the chunks, their creation, and the ability of the brain to store and execute chunks in the neocortex (creation and establishment of skills) are what determines giftedness for a particular task and that they are the key factor in the creation of expert memory, and not just that of chess players.

\section{References}

Amidzic, O., Riehle, H.J., Fehr, T., Wienbruch, C., \& Elbert, T. (2001). Pattern of focal $\gamma$-bursts in chess players. Nature, 412, 603.

Bontempi, B., Laurent-Demir, C., Destrade, C., \& Jaffard, R. (1999). Time-dependent reorganization of brain circuitry underlying long-term memory storage. Nature, 400, 671-674.

Braitenberg, V., \& Schüz, A. (1991). Anatomy of the cortex. Berlin: Springer.

Chase, W.G., \& Simon, H.A. (1973a). The mind's eye in chess. In W.G. Chase (Ed.), Visual information processing (pp. 215281). New York: Academic Press.

Chase, W.G., \& Simon, H.A. (1973b). Perception in chess. Cognitive Psychology, 4, 51-81.

Elbert, T. (1998). Neuromagnetism. In W. Andrä \& H. Nowak (Eds.), Magnetism in medicine (pp. 190-262). Berlin: Wiley.

Elo, A.E. (1978). The rating of chessplayers, past and present. New York: Arco.

Ericsson, K.A., \& Kintsch, W. (1995). Long-term working memory. Psychological Review, 102, 211-245.

Fernandez, A., Maestu, F., Amo, C., Gil, P., Fehr, T., Wienbruch, C., Rockstroh, B., Elbert, T., \& Ortiz, T. (2002). Focal temporoparietal slow activity in Alzheimer's disease revealed by magnetoencephalography. Biological Psychiatry, 52, 764770 .

Gobet, F. (1998). Expert memory: A comparison of four theories. Cognition, 66, 115-152.

Gobet, F., \& Simon, H.A. (1996a). Recall of random and distorted chess positions: Implications for the theory of expertise. Memory \& Cognition, 24, 493-503.

Gobet, F., \& Simon, H.A. (1996b). Templates in chess memory: A mechanism for recalling several boards. Cognitive Psychology, 31, 1-40. 
Holding, D.H. (1992). Theories of chess skill. Psychological Research, 54, 10-16.

Holding, D.H., \& Reynolds, R.I. (1982). Recall or evaluation of chess positions as determinants of chess skill. Memory \& Cognition, 10, 237-242.

Lewine, J.D., \& Orrison, W.W. (1995). Magnetoencephalography and magnetic source imaging. In W.W. Orrison, J.D. Lewine, J.A. Sanders, \& M.F. Hartshorne (Eds.), Functional brain imaging (pp. 369-417). St. Louis: Mosby.

Meinzer, M., Elbert, T., Wienbruch, C., Djundja, D., Barthel, G., \& Rockstroh, B. (2004). Intensive language training enhances brain plasticity in chronic aphasia. BMC Biology, 2, 20.

Pulvermüller, F., Keil, A., \& Elbert, T. (1999). High-frequency brain activity: Perception or active memory? Trends in Cognitive Sciences, 3, 250-252.

Rioult-Pedotti, M.S., Friedman, D., \& Donoghue, J.P. (2000). Learning-induced LTP in neocortex. Science, 290, 533-535.

Rockstroh, B., Ray, W., Wienbruch, C., \& Elbert, T. (2004). Identification of dysfunctional cortical network architecture and communication: Abnormal slow wave activity mapping (ASWAM) in neurological and psychiatric disorders. Submitted for publication.

Singer, W., Engel, A.K., Kreiter, A.K., Munk, M.H.J., Neuenschwander, S., \& Roelfsema, P.R. (1997). Neuronal assemblies: Necessity, signature, and detectability. Trends in Cognitive Sciences, 1, 252-261.

Squire, L.R. (1992). Memory and the hippocampus: A synthesis from findings with rats, monkeys, and humans. Psychological Revue, 99, 195-231.

Tallon-Baudry, C., \& Bertrand, O. (1999). Oscillatory gamma ac- tivity in humans and its role in object representation. Trends in Cognitive Sciences, 3, 151-162.

Teng, E., \& Squire, L.R. (1999). Memory for places learned long ago is intact after hippocampal damage. Nature, 400, 675-677.

Vieth, J.B., Kober, H., \& Grummich, P. (1996). Sources of spontaneous slow waves associated with brain lesions, localized by using the MEG. Brain Topography, 8, 215-221.

Wienbruch, C., Moratti, S., Elbert, T., Vogel, U., Fehr, T., Kissler, J., et al. (2003). Source distribution of neuromagnetic slow wave activity in schizophrenic and depressive patients. Clinical Neurophysiology, 114, 2052-2060.

Zola-Morgan, S.M., \& Squire, L.R. (1990). The primate hippocampal formation: Evidence for a time-limited role in memory storage. Science, 250, 288-290.

Ognjen Amidzic

Universität Konstanz

Sportwissenschaft

Postfach D 30

Universitätsstraße 10

D-78457 Konstanz

Germany

Tel. +41793872737

Fax +49 $753188-4221$

E-mail ognjen.amidzic@uni-konstanz.de 